\title{
A study of the regular structure of the galactic magnetic field using WMAP5 polarization data at $22 \mathrm{GHz}$
}

\author{
Beatriz Ruiz-Granados ${ }^{1}$, J. A. Rubiño-Martín ${ }^{2}$ \\ and E. Battaner ${ }^{1}$ \\ ${ }^{1}$ Dpto. Física Teórica y del Cosmos. Edif. Mecenas. Campus Fuentenueva, E-18071. \\ Universidad de Granada \& Instituto de Física Teórica y Computacional Carlos I, Granada \\ (Spain) \\ email: bearg@ugr.es, battaner@ugr.es \\ ${ }^{2}$ Instituto de Astrofísica de Canarias (IAC), C/Vía Láctea, s/n, E-38200, La Laguna (Spain) \\ email: jalberto@iac.es
}

\begin{abstract}
We study the spatial structure of the 3-dimensional large-scale pattern of the Galactic Magnetic Field using the polarization maps obtained by the WMAP satellite at $22 \mathrm{GHz}$. By using five different models of the large-scale magnetic field of the Milky Way and a model for the cosmic rays distribution, we predict the expected polarized synchrotron emission. Those maps are compared to the observed $22 \mathrm{GHz}$ polarization data using a Maximum Likelihood method. For each model, we obtain the parameter values which better reproduce the data and obtain their marginal probability distribution functions. We find that the model that best reproduces the observed polarization maps is an "axisymmetric" model.
\end{abstract}

Keywords. Magnetic fields - galaxies: structure - polarization

\section{Model of the galactic magnetic field}

Since the discovery of the polarized synchrotron emission from our Galaxy (Wielebinski \& Shakeshaft 1962), several works have attempted to constrain the pattern of the regular component of the Galactic Magnetic Field (GMF). Recent studies of the GMF are given in Sun et al. (2008), Noutsos et al. (2008) and Men et al. (2008). At 22 GHz, the physical mechanism which produces the dominant contribution to the large-scale pattern of the radio-emission is the synchrotron radiation. Two ingredients are needed to predict the polarized synchrotron emission of the Galaxy. The first one is a model describing the cosmic rays electron distribution; in this work, we use the model by Drimmel \& Spergel (2001). The second ingredient is a model for the regular component of the GMF. Here we have explored five different models, namely: (a) Axisymmetric Spiral (ASS; with and without radial dependence of the strength); (b) Logarithmic Spiral Arms (LSA; as proposed by Page et al. 2007), (c) Bi-symmetric Spiral (BSS) with and without radial dependence of the strength (Han \& Qiao (1994)); (d) Concentric Circular Ring (CCR; Rand \& Kulkarni 1989); and (e) Bi - Toroidal (BT; based on Han 1997).

\section{Method}

For every considered model, we simulate the Stokes's U and Q parameters and the polarization angle (PA) following the procedure described in Ruiz-Granados et al. (in prep). The basic assumption is that the dominant emission process is synchrotron with an average spectral index of $\sim-2.7$. For each model, we perform a blind exploration 

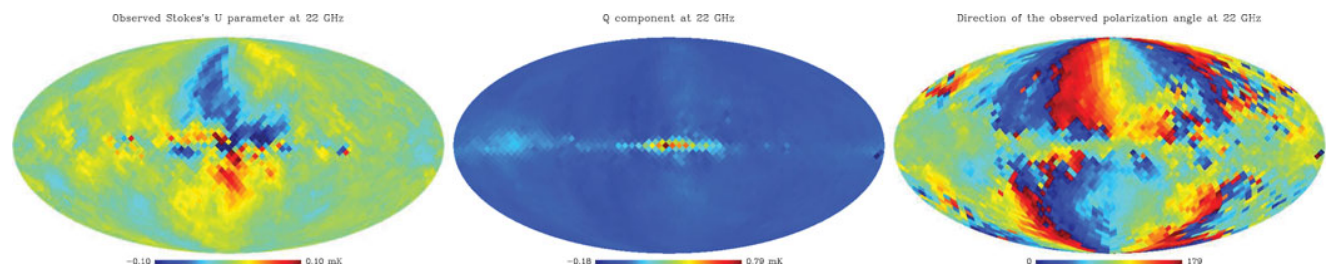

Figure 1. Observed U, Q and PA maps at $22 \mathrm{GHz}$ (WMAP 5-year maps).
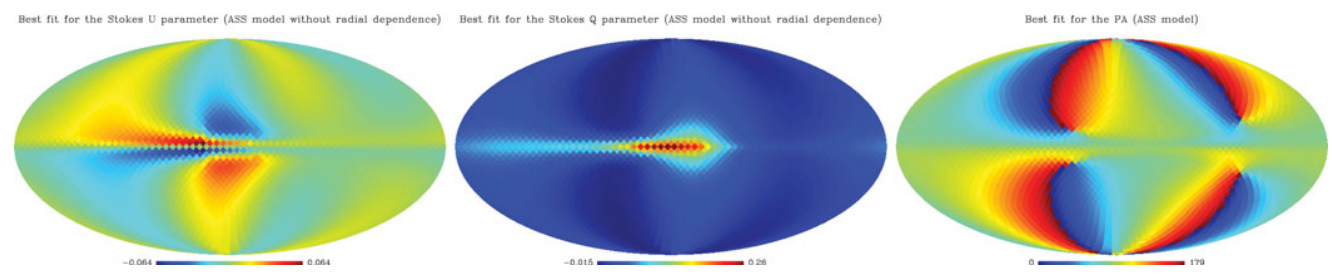

Figure 2. Predicted $22 \mathrm{GHz}$ U, Q and PA maps from our best-fit model for the GMF.

of the parameter space by obtaining the $\chi^{2}$ statistics both from the $(Q, U)$ and PA maps. Using a Bayesian approach, we build the likelihood function as $\ln L=-1 / 2 \chi^{2}$, and compute the marginalized posteriors for each parameter. The comparison between different models is done using the reduced $\chi^{2}$ as the "goodness-of-fit" statistic.

\section{Conclusions}

The model that best reproduces the large-scale pattern of the observed WMAP polarization data at $22 \mathrm{GHz}$ is ASS without any radial dependence of the strength of the field. The parameter values for the best-fit are: $B_{0}=\left(2.5 \pm_{0.8}^{1.7}\right) \mu \mathrm{G}$, pitch angle $p=\left(24 \pm_{0.8}^{0.8}\right)^{\circ}$ and tilt angle $\chi_{0}=\left(24 \pm_{1.3}^{1.2}\right)^{\circ}$. Fig. 1 shows the observed U, Q and PA maps, while Fig. 2 presents the corresponding simulated maps for the best-fit model. We note that the best-fit model is obtained in all cases when masking the loops (the NPS and other spurs) and the Galactic center region, due to the disturbance of the emission introduced by these regions with respect to the regular pattern.

In general, all axisymmetric-like models considered here provide reasonable fits. In particular, the best-fit LSA model practically coincides with the ASS one, since the data do not seem to require a radial variation of the pitch angle. Both the BSS, CCR and BT models do not provide good fits to the data. However, the BT could reproduce the halo component.

\section{References}

Drimmel, R. \& Spergel, D. N. 2001, ApJ, 556, 181

Han, J. L. \& Qiao, G. J. 1994 A\&SA 288, 759

Han, J.-L. 1997 Chinese Astronomy and Astrophysics 21, 130

Men, H., Ferriere, K., \& Han, J. L. 2008 A $\& A$ 486, 819

Noutsos, A., Johnston, S., Kramer, M., \& Karastergiou, A. 2008 MNRAS 386, 1881

Page, L., et al. 2007 ApJ. Suppl. 170, 335

Rand, R. J. \& Kulkarni, S. R. 1989 ApJ. 343, 760

Sun, X. H., Reich, W., Waelkens, A., \& Enßlin, T. A. 2008 A $\& A 477,573$

Wielebinski, R. and Shakeshaft, J. R. 1962 Nature 195, 982 

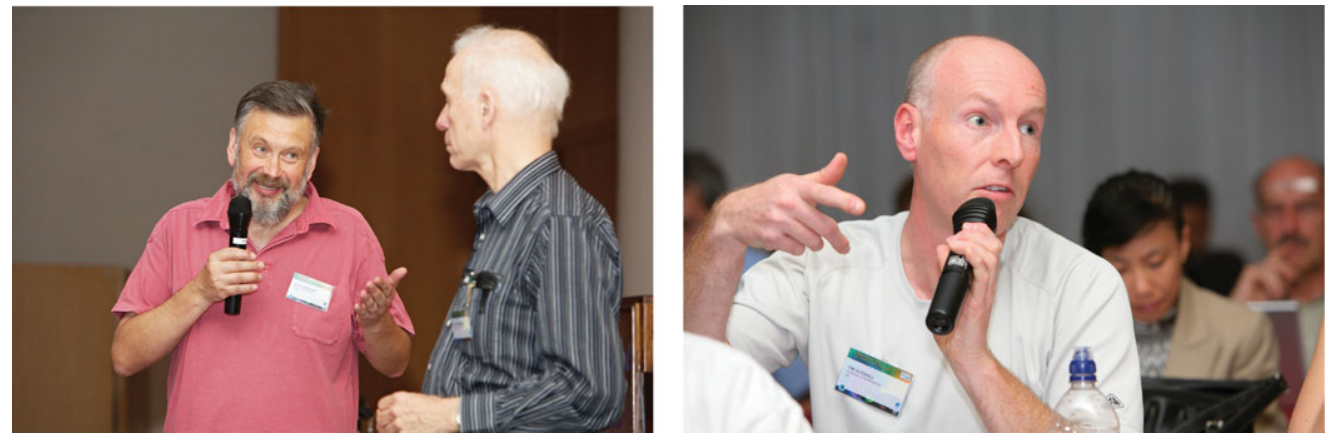

Session chair Dimitry Sokoloff arguing with Philipp Kronberg (left), and Tim Gledhill (right)
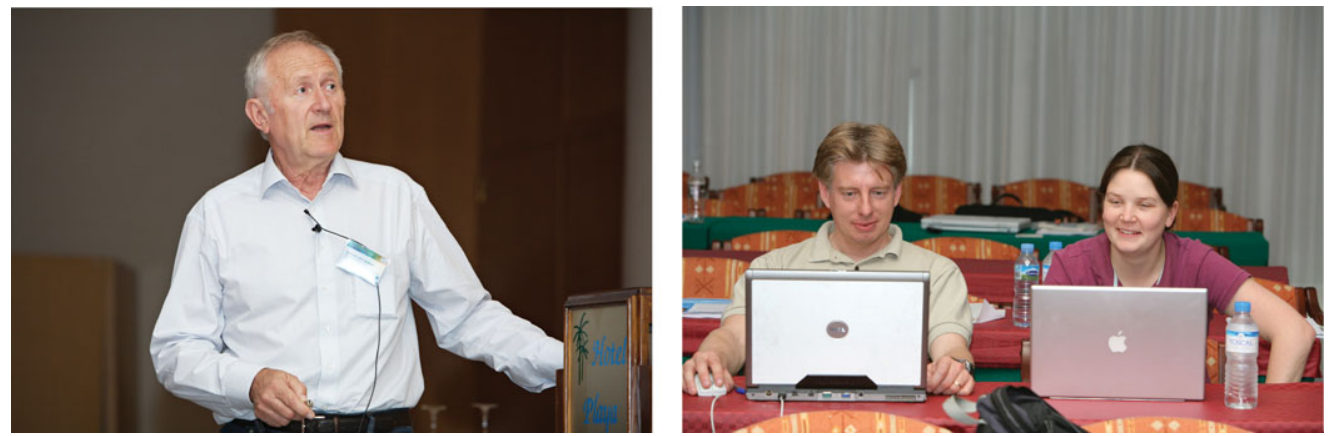

Richard Wielebinski (left), and Jeroen Stil \& Julie Grant (right)
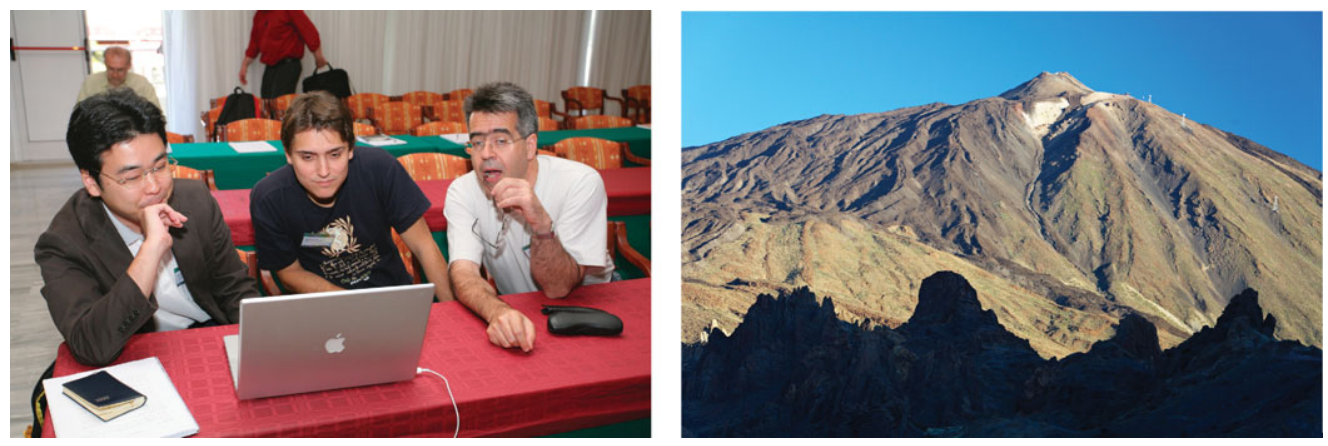

Ryo Kandori, Felipe Alves \& Gabriel Franco (left to right), and the Teide (picture by R. Arlt)
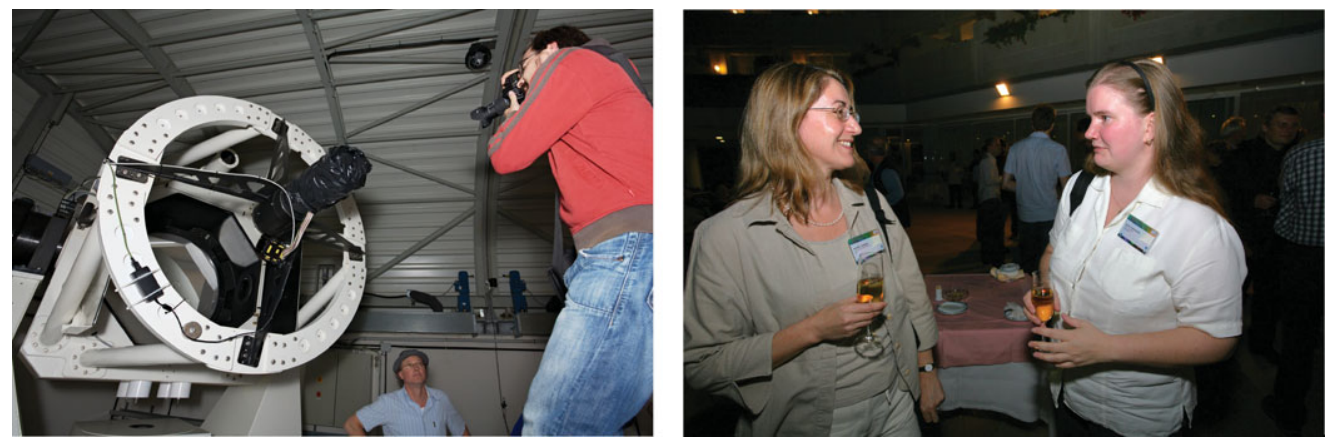

Visit at STELLA (left), and Moira Jardine \& Heidi Korhonen (right) 


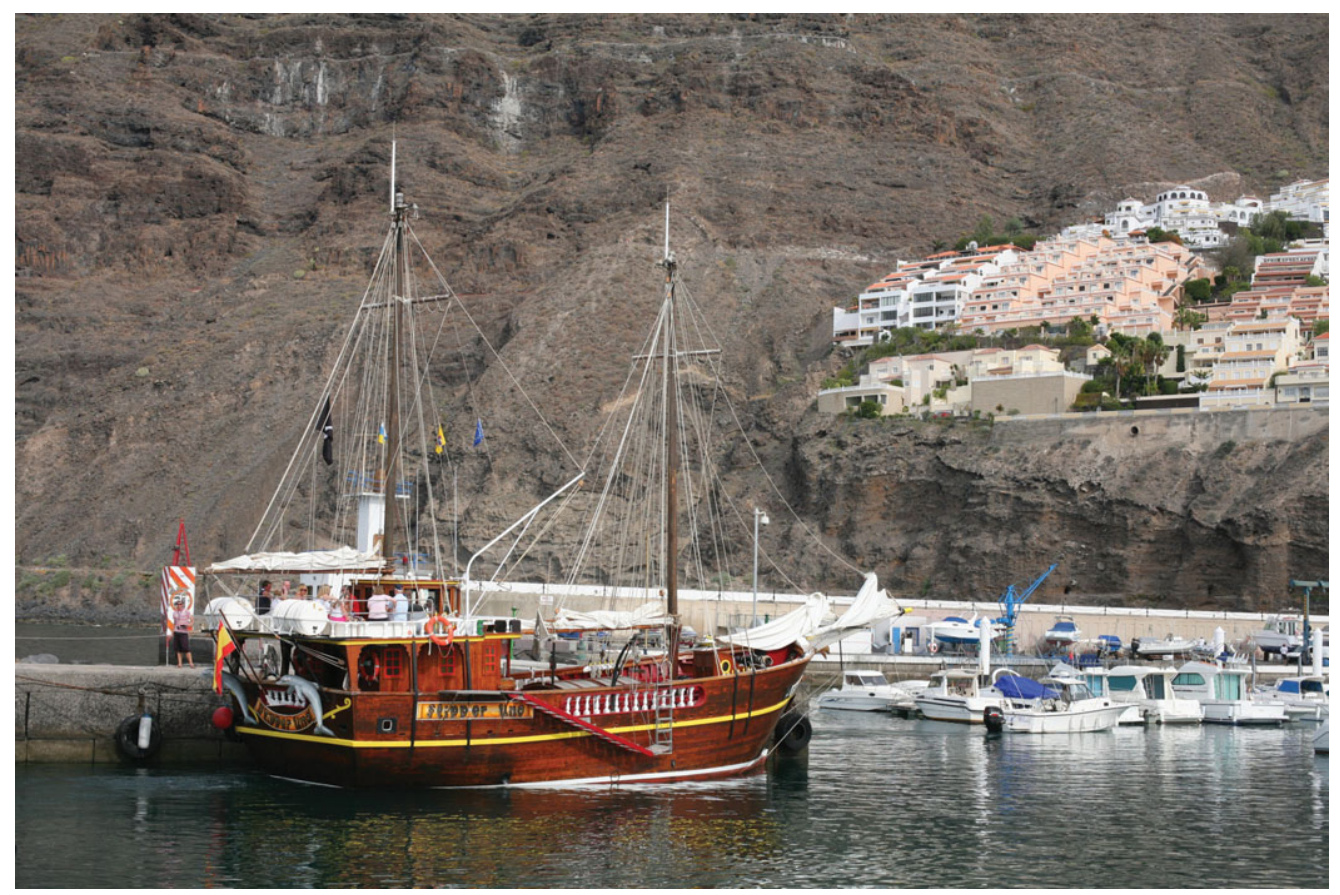

Ready to leave the harbor with the "pirates" ship

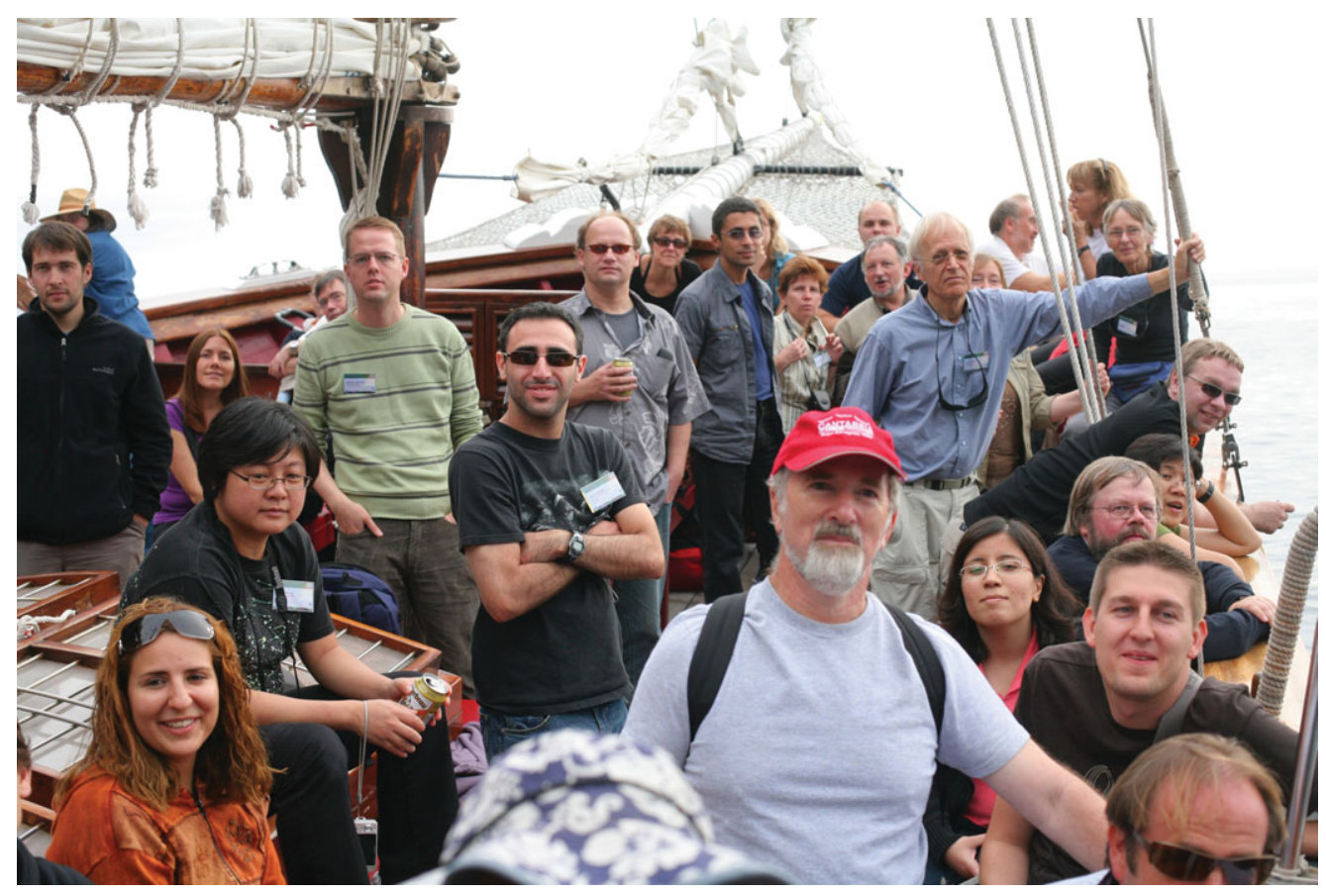

The crew 